University of Louisville

ThinkIR: The University of Louisville's Institutional Repository

\title{
Structural and functional neural correlates of a mind-body connection.
}

Leonard Faul

University of Louisville

Follow this and additional works at: https://ir.library.louisville.edu/honors

Part of the Health Psychology Commons

\section{Recommended Citation}

Faul, Leonard, "Structural and functional neural correlates of a mind-body connection." (2017). College of Arts \& Sciences Senior Honors Theses. Paper 141.

http://doi.org/10.18297/honors/141

This Senior Honors Thesis is brought to you for free and open access by the College of Arts \& Sciences at ThinkIR: The University of Louisville's Institutional Repository. It has been accepted for inclusion in College of Arts \& Sciences Senior Honors Theses by an authorized administrator of ThinkIR: The University of Louisville's Institutional Repository. This title appears here courtesy of the author, who has retained all other copyrights. For more information, please contact thinkir@louisville.edu. 
Structural and Functional Neural Correlates of a Mind-Body Connection

By

\section{Leonard Faul}

Submitted in partial fulfillment of the requirements for Graduation summa cum laude

and

for Graduation with Honors from the Department of Psychological and Brain Sciences

University of Louisville

March, 2017 


\begin{abstract}
The human brain underlies the complex cognitive processes of the mind, however, this is dependent upon the physiological processes of the body in order to receive adequate energy, oxygen, and blood flow. Therefore, physical measurements such as body mass index (BMI) and indices of cognitive functioning, such as intelligence, may be related via common neural features. Current analyses assessed morphometric differences in cortical and subcortical grey matter regions, white matter structural integrity, and resting-state functional activation in order to determine what combinations of neural variables predict BMI and intelligence (Wechsler Abbreviated Scale of Intelligence; WASI) with the best degree of accuracy. Data for eighty-five subjects was obtained from the Nathan Kline Institute, in connection with the 1000 Functional Connectomes neuroimaging database. Behavioral results indicated a negative correlation between BMI and WASI scores. Neural analyses revealed that increased BMI predicted changes in a frontolimbic network comprised of the anterior cingulate cortex, amygdala, and uncinate fasciculus, as well as increased cortical surface area of the left fusiform gyrus. These results indicate a relationship of BMI with emotional decision-making and visual recognition processes. Whereas, increased WASI scores predicted increased thickness and volume of prefrontal and parietal cortices, which reflect brain regions involved in the fronto-parietal attentional network. As well, increased WASI scores also related to a functional network that included increased activation of the postcentral gyrus and posterior hippocampal complex, regions involved with attention and memory. Taken together, these results indicate that BMI and intelligence are behaviorally anticorrelated, yet mediated by separate neuroanatomical substrates that associate with a variety of cognitive functioning measures.
\end{abstract}

Keywords: BMI, intelligence, attention, morphometry, white matter, resting-state 


\section{Introduction}

Dating back millennia, philosophers such as Socrates and Aristotle have debated the relationship between the mind and the body. How does one relate physical matter to the mental processes that allow us to think and to feel? Current philosophers and scientists still debate and investigate this same dilemma in order to understand the underlying basis of consciousness. However, today we also recognize the importance of a neural link between the mind and the body, thereby extending the mind-body relationship to a mind-brain-body connection.

Understandably, the brain is considered to be the most complex organ in the human body. It is the culmination of multiple structural and functional neural features that allows for an efficient cognitive system to operate. The field of cognitive neuroscience has consistently studied the relationship between the brain and behavior in an effort to better understand the importance and utilization of different brain regions and, more importantly, wide spread neural networks. During the last two decades researchers have also come to increasingly acknowledge the inter-reliance and dependency of the brain and body. The human brain underlies the complex cognitive processes of the mind, however, this is dependent upon the physiological processes of the body in order to receive adequate energy, oxygen, and blood flow.

There are two main questions that the present study is aimed to address. First, how do physiological measures of the body and cognitive measures of the mind influence brain structure and function? Second, how do common neural features help explain the relationship between cognitive functioning and body physiology? We therefore examine the association between a general indicator of body health and a general indicator of 
cognitive functioning in order to demonstrate the importance of a neural connection between the two measures. Few studies have pursued such a comprehensive brain-mindbody investigation to understand cognitive functioning, yet we argue that such an approach may elucidate important relationships and reveal interactions that otherwise would be overlooked.

How do physiological measures of the body influence brain structure and function?

In order to analyze the relationship between the body and brain, most studies investigate the effects of physiological dysfunction and the corresponding associations with neural features. Research concerning obesity best exemplifies this, since weight gain is known to accompany a wide range of health complications, including high blood pressure and cholesterol levels, arthritis, diabetes, and cancer (Mokdad et al., 2003; Kopelman 2007). Obesity induces heightened inflammation among adipocytes, which subsequently causes metabolic, immune and other homeostatic physiological processes to lose their normal functioning (Greenberg \& Obin, 2006; Lee \& Lee, 2014).

Although obesity can be classified based on a variety of measurements, the World Health Organization (WHO) has used body mass index (BMI) as a "crude population measure of obesity," which is calculated as kilograms per square meter and therefore incorporates both the height and weight of an individual (Keys et al., 1972). Typically, those with a BMI less than 18.5 are considered to be underweight, 18.5 - 24.99 normal or healthy, $25-29.99$ overweight, and those with a BMI greater than or equal to 30 obese.

With such a wide range of health implications, it is not surprising that obesity has significant relationships with both brain structure and function. Previous studies examining this relationship show that high BMI measurements predict smaller whole 
brain and total grey matter volume (Gunstad et. al., 2008; Boyle et. al 2015). Obese individuals also tend to show reduced cortical thickness in frontal (dorsal anterior cingulate cortex $-\mathrm{dACC}$ - and medial orbitofrontal cortex) and parietal (posterior parietal cortex) regions, when compared to non-obese controls (Hassenstab et al., 2012; Marques-Iturria et al., 2013). Furthermore, obesity is associated with reduced fractional anisotropy (FA) - a measure of white matter structural integrity - in the corpus callosum and fornix (Stanek et al., 2011), as well as differences in cognitive control and reward networks when assessed with functional magnetic resonance imaging (fMRI) during resting-state (Kullmann et al., 2012; Lips et al., 2014).

It is worth emphasizing, however, that BMI is an indirect measure of body physiology and therefore is limited in its ability to truly classify an individual as overweight or obese. It is useful then to also investigate individual variations in neural features across a broad range of BMI measures, as opposed to grouping all individuals in either high or low BMI, in order to determine correlational relationships with the brain. Regarding specific brain morphometry, a recent study found that increased BMI predicts decreased cortical thickness in the left lateral occipital cortex and the right ventromedial prefrontal cortex - vmPFC (Medic et al., 2016). Similarly, increases in BMI over a oneyear period correlate with decreases in cortical volume of the superior and middle frontal gyri among female adolescents (Yokum et al., 2012). Regarding white matter structural integrity, increasing BMI also predicts decreasing FA and structural integrity of the corpus callosum and fornix (Xu et al., 2013). Therefore, even when looking at individual differences in BMI, including those with a healthy BMI range, similar negative relationships as group analyses are observed between BMI and brain morphometry. 


\section{How do cognitive measures of the mind influence brain structure and function?}

Intelligence is arguably one of the most notable measures of cognitive functioning, given its long history of association with social status, education, and a wide variety of other performance measures (Neisser et al., 1996; Gottfredson, 1998). Charles Spearman first introduced the concept of general intelligence $(g)$ to explain how individuals could consistently display high levels of cognitive ability on multiple different assessments (Spearman, 1904). This also set the stage for the intelligence quotient (IQ), a number that quantifies performance on these tests and therefore functions to measure one's intellectual ability.

Just as BMI serves as a general indicator of the body, intelligence also serves as a general indicator of the mind. The evolution of the human brain is closely tied to increases in intelligence as an indicator of progressively more complex and integrated cognitive functioning (Roth \& Dicke, 2005). As well, intelligence scores can increase within an individual following training with working-memory tasks (Jaeggi et al., 2008; Au et al., 2015), emphasizing its representation of dynamic cognitive ability that can be enhanced over time.

In regards to a neural relationship, increasing intelligence generally has a positive association with whole brain grey and white matter volume (Gignac et al., 2003; Haier et al., 2004). Increasing intelligence is also generally associated with broad increases in cortical thickness across frontal and parietal regions (Menary et al., 2013), as well as increased functional connectivity in neural networks incorporating these same regions (Song et al., 2007). These findings seem to contrast with the effects of increasing BMI, especially in regard to structural differences. This raises the possibility that higher 
cognitive functioning ability, such as increasing intelligence, may associate with lower BMI.

How do common neural features help explain the relationship between cognitive functioning and body physiology?

Overweight and obese individuals show poorer performance on intelligence and executive functioning tests, as compared to other adults in a normal BMI range (Gunstad et al., 2007; Kanazawa, 2014). Furthermore, it has also been shown that a relationship between increased BMI and decreased cortical thickness in the right superior frontal gyrus is mediated by reduced cognitive control (Lavagnino et. al., 2016). Perhaps a similar brain-mind-body connection can be made between BMI and intelligence.

Therefore, the present study sought to investigate the relationship between BMI (a general measure of the body) and intelligence (a general measure of the mind) and subsequently explore a wide variety of neural features that may help explain this relationship. We assessed cortical and subcortical grey matter morphometry as well as the integrity of various white matter tracts in order to identify morphometric features that relate to BMI and intelligence measures. In addition, we analyzed behaviorally relevant intrinsic connectivity networks using resting-state fMRI analysis. It was hypothesized that BMI and intelligence would show a negative relationship with one another, as a primary consequence of contrasting associations with cortical structures and divergent effects on intrinsic resting-state networks.

\section{Methods}

The data used in this investigation were obtained from the 1000 Functional Connectomes Project (FCP) database. The FCP was created in 2009 and works with the 
International Neuroimaging Data-Sharing Initiative (INDI) to provide public access to large datasets, all of which include resting-state fMRI data. The Nathan Kline Institute Rockland Sample (NKI-RS) is a prospectively shared dataset that offers data from individuals between 4 to 85 years old and includes a wide range of phenotypic information for all of their subjects, as well as anatomical (MPRAGE), diffusion weighted imaging (DWI), and resting-state fMRI scans.

\section{Participants}

A total of 85 adults (55 males, 30 females), $18-59$ years of age $(\mathrm{M}=33.5, \mathrm{SD}=$ 11.0) were included in this study. Two subjects were missing total IQ scores and one subject was missing a BMI measurement. In order to increase power in the neural analyses, these individuals were provided with the average scores of the sample.

Neuroimaging data from all individuals were used in the surface-based morphometry, TBSS white matter, and resting-state fMRI analyses. One subject was removed from the FIRST subcortical analysis due to unsatisfactory subcortical segmentation.

\section{Behavioral Assessments}

Of primary interest for this study were BMI measurements and scores on the Wechsler Abbreviated Scale of Intelligence (WASI). BMI is calculated by dividing the weight of an individual by the square of their height and is therefore reported in $\mathrm{kg} / \mathrm{m}^{2}$. The World Health Organization (WHO) considers a BMI of less than 18.5 as underweight, $18.50-24.99$ as normal, $25.00-29.99$ as overweight, and anything above 30 as obese.

The WASI-II is an abbreviated measure of cognitive intelligence and is comprised of four main subtests given in the following order: block design, vocabulary, matrix 
reasoning, and similarities, (Wechsler, 2011). Block design and matrix reasoning form the perceptual reasoning index (PRI), whereas vocabulary and similarities form the verbal comprehension index (VCI). During the block design test subjects must recreate a twodimensional geometric design using cubes, thereby testing fluid intelligence and visual perception. Similarly, the matrix reasoning test also evaluates fluid and visual intelligence by requiring examinees to complete a matrix by selecting the appropriate choice among a set of options. In contrast, the vocabulary test measures verbal, crystallized intelligence and does so by instructing the examinee to define a word or concept that is presented to them, whereas the similarities test evaluates similar abilities by requiring the subject to describe the relationship between two objects or concepts. The NKI Rockland Sample data included a full WASI score, as well as scores on each individual subtest. Correlational analyses were assessed with SPSS 22.0 software (IBM Corp, Somers, NY, USA) in order to determine the relationship between BMI and WASI scores, while controlling for age.

\section{Imaging Data Acquisition}

Scans were acquired using a Siemens Tim Trio 3T scanner with a 12-channel head coil. Imaging parameters for structural MRI images were as follows: voxel size 1.0 x $1.0 \times 1.0 \mathrm{~mm}$, repetition time (TR) $2500 \mathrm{~ms}$, echo time (TE) $3.5 \mathrm{~ms}$. Imaging parameters for diffusion images were as follows: voxel size $2.0 \times 2.0 \times 2.0 \mathrm{~mm}$, TR $10000 \mathrm{~ms}$, TE $91 \mathrm{~ms}, \mathrm{~b}$-value $1000 \mathrm{~s} / \mathrm{mm}^{2}$. Imaging parameters for functional MRI images were as follows: voxel size $3.0 \times 3.0 \times 3.0 \mathrm{~mm}$, TR $2500 \mathrm{~ms}$, TE $30 \mathrm{~ms}, 260$ volumes.

For more information, visit http://fcon_1000.projects.nitrc.org/indi/pro/nki.html. Surface-Based Morphometry (SBM) 
Cortical reconstruction and volumetric segmentation was performed with the Freesurfer image analysis suite, which is documented and freely available for download online (http://surfer.nmr.mgh.harvard.edu/). The technical details of these procedures are described in prior publications (Dale, Fischl, \& Sereno, 1999). Briefly, this processing includes motion correction and averaging (Reuter, Rosas, \& Fischl, 2010) of volumetric T1-weighted images, removal of non-brain tissue using a hybrid watershed/surface deformation procedure (Ségonne et al., 2004), automated Talairach transformation, intensity normalization (Sled, Zijdenbos, \& Evans, 1998), tessellation of the gray matter white matter boundary, automated topology correction (Fischl, Liu, \& Dale, 2001; Segonne, Pacheco, \& Fischl, 2007), and surface deformation following intensity gradients to optimally place the gray/white and gray/cerebrospinal fluid borders at the location where the greatest shift in intensity defines the transition to the other tissue class (Dale et al., 1999; Dale \& Sereno, 1993; Fischl \& Dale, 2000). Once the cortical models are complete, a number of deformable procedures are performed for further data processing and analysis including surface inflation (Fischl, Sereno, \& Dale, 1999), registration to a spherical atlas which utilizes individual cortical folding patterns to match cortical geometry across subjects (Fischl, Sereno, Tootell, \& Dale, 1999), parcellation of the cerebral cortex into units based on gyral and sulcal structure (Desikan et al., 2006; Fischl et al., 2004), and creation of a variety of surface-based data including maps of cortical volume, surface area, thickness, curvature, sulcal depth, and local gyrification index. The resulting probability maps were input into a general linear model (GLM) evaluating regressions between all voxels and BMI or WASI scores, controlling for age and whole-brain intracranial volume (ICV). Vertex-wise threshold was set at $\mathrm{p}<.001$ 
level. Cluster-wise threshold was corrected for at $\mathrm{p}<.05$ level using permutation testing with Monte Carlo simulation.

FIRST Subcortical Vertex Analysis

Anatomical imaging data was segmented into subcortical regions, using FSL's FMRIB Integrated Registration and Segmentation Tool (FIRST; FSL v5.0.9, http://www.fmrib.ox.ac.uk/fsl/first/index.html), which is a model-based segmentation tool that allows for parcellation of several subcortical brain structures from high resolution T1- weighted images of the brain (Patenaude, 2007; Patenaude, Smith, \& Kennedy, 2007, 2008). Importantly, this tool enables an unbiased approach to segmentation of the regions, which are notoriously hard to manually trace due to their small structure size.

Automated segmentation of 15 subcortical structures (Brain Stem and bilateral: Accumbens, Amygdala, Caudate, Hippocampus, Putamen, Pallidum, and Thalamus) was performed using FIRST (FSL v5.0.9) which uses a Bayesian probabilistic approach. The shape and appearance models in FIRST are constructed from a library of manually segmented images. The manually generated labels are parameterized as surface meshes and then modeled as a point distribution. Using the learned models, FIRST searches through shape deformations that are linear combinations of the modes of variation to find the most probable shape instance given the observed intensities from the input image. Using T1 images, the segmentation was performed with two-stage affine transformation to standard space of MNI 152 at 1-mm resolution (Morey et al., 2009; Woolrich et al., 2009). The first stage utilized a standard 12 degrees of freedom registration to the template and the second stage applied 12 degrees of freedom registration using an 
MNI152 subcortical mask to exclude voxels outside the subcortical regions. Default settings for boundary correction and number of modes (iterations) were used, which are tuned to be optimal for each structure. All segmentations were then visually inspected by two independent viewers to ensure satisfactory segmentation of all structures of interest. Volume was also extracted for each structure. Shape and volume regressions were then performed to investigate differences in localized shape on a per-vertex basis and total volume of a structure, with BMI and WASI scores. All analyses included age and ICV as nuisance covariates. FSL's randomise tool was used to calculate statistics from the image output after 5000 permutations (Winkler et al., 2014) and threshold-free cluster enhancement (TFCE), with significance set at $p<0.05$.

Tract-Based Spatial Statistics (TBSS) Analysis

Voxelwise statistical analysis of diffusion data was carried out using TBSS (Smith, 2006), a tool provided by FSL (Smith, 2004). First, diffusion weighted images were corrected for eddy-current-induced distortions. Fractional anisotropy (FA) images were then created by fitting a tensor model to the raw diffusion data using FSL's Diffusion Toolbox (FDT), and then brain-extracted using BET [Smith 2002]. All subjects' FA data were then aligned into a common space using the nonlinear registration tool FNIRT [Andersson 2007, 2007], which uses a b-spline representation of the registration warp field [Rueckert 1999]. Next, the mean FA image was created and thinned to create a mean FA skeleton, which represents the centres of all tracts common to the group. Each subject's aligned FA data was then projected onto this skeleton and the resulting data fed into voxelwise cross-subject statistics at $p<.001$. We then tested for individual differences relating to BMI and WASI using FSL's randomise permutation- 
testing tool, with 5000 permutations controlling for age and using TFCE to correct for multiple comparisons.

Functional Analysis

Resting-state fMRI data was assessed using independent components analysis (ICA), via FSL's MELODIC (Multivariate Exploratory Linear Optimized Decomposition into Independent Components) tool, version 3.14 (Beckmann \& Smith, 2004). This separates the functional activation into a set of spatiotemporal components, which can then be analyzed as intrinsic networks of interest. Using FSL, resting-state fMRI scans were motion corrected, brain extracted, and set through a smoothing process. The functional data was first registered to subject-specific anatomical volumes, followed by registration to MNI152 standard space.

Multi-session temporal concatenation identified common spatial patterns among the subjects, while accounting for the inconsistency of the temporal responses. The total number of output components was set to 30 , which were statistically compared to seven popular resting-state networks (Yeo et al., 2011). 12 components were considered to represent distinct networks after showing significant spatial correlations (Pearson's r > $0.207, p=.05)$ with the reference networks.

The set of spatial maps from the group-average analysis were then used to generate subject-specific versions of the spatial maps, and associated timeseries, using dual regression (Beckmann, 2009; Filippini, 2009). First, for each subject, the groupaverage set of spatial maps was regressed (as spatial regressors in a multiple regression) into the subject's 4D space-time dataset. This resulted in a set of subject-specific timeseries, one per group-level spatial map. Next, those timeseries were regressed (as 
temporal regressors, again in a multiple regression) into the same $4 \mathrm{D}$ dataset, resulting in a set of subject-specific spatial maps, one per group-level spatial map. We then tested for individual differences relating to BMI and WASI using FSL's randomise permutationtesting tool, with 5000 permutations controlling for age and using TFCE to correct for multiple comparisons.

\section{Results}

\section{Behavioral Results}

The correlation between full WASI score and BMI was negatively correlated $(r=$ $-0.288, p=0.008$ ), such that increased intelligence was related to decreased BMI (Figure 1). This same negative relationship was also found for each subtest, however, only significant for the vocabulary and similarities tests (Table 1).

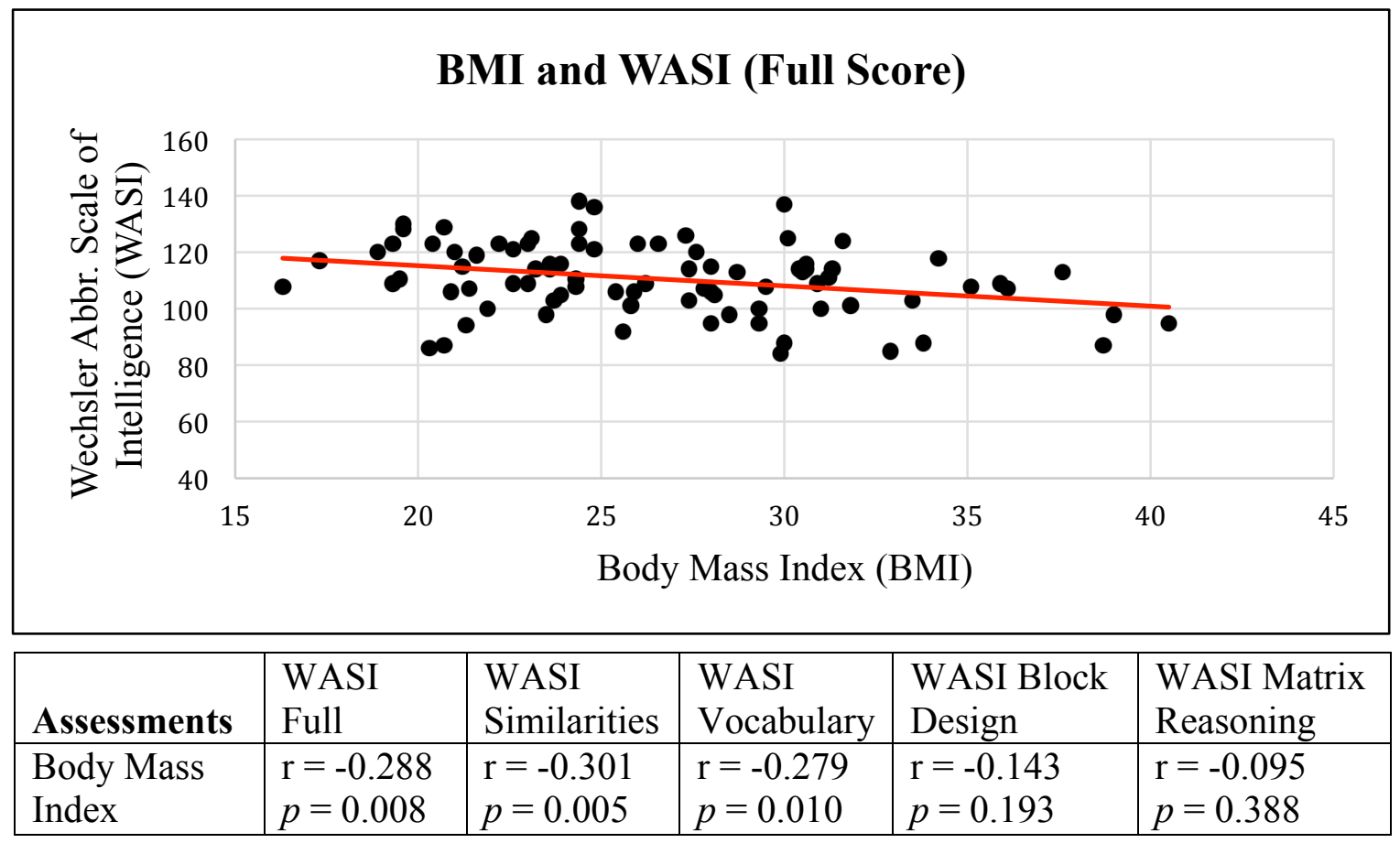

Figure 1: shows the correlation of BMI and full WASI scores across all subjects. Table 1 reports the correlation coefficient and significance for this relationship, as well as for the relationship between BMI and each WASI subtest. 


\section{Body Mass Index (BMI) Results}

Surface-Based Morphometry (SBM)

Regressions with cortical grey matter morphometry indicate that increased BMI predicted decreased cortical thickness in the left anterior cingulate cortex (ACC) and part of the ventromedial prefrontal cortex (vmPFC), as well as increased surface area in the left fusiform gyrus (Figure 2), suggesting that increased body mass had both an increased and decreased effect on cortical morphometry.
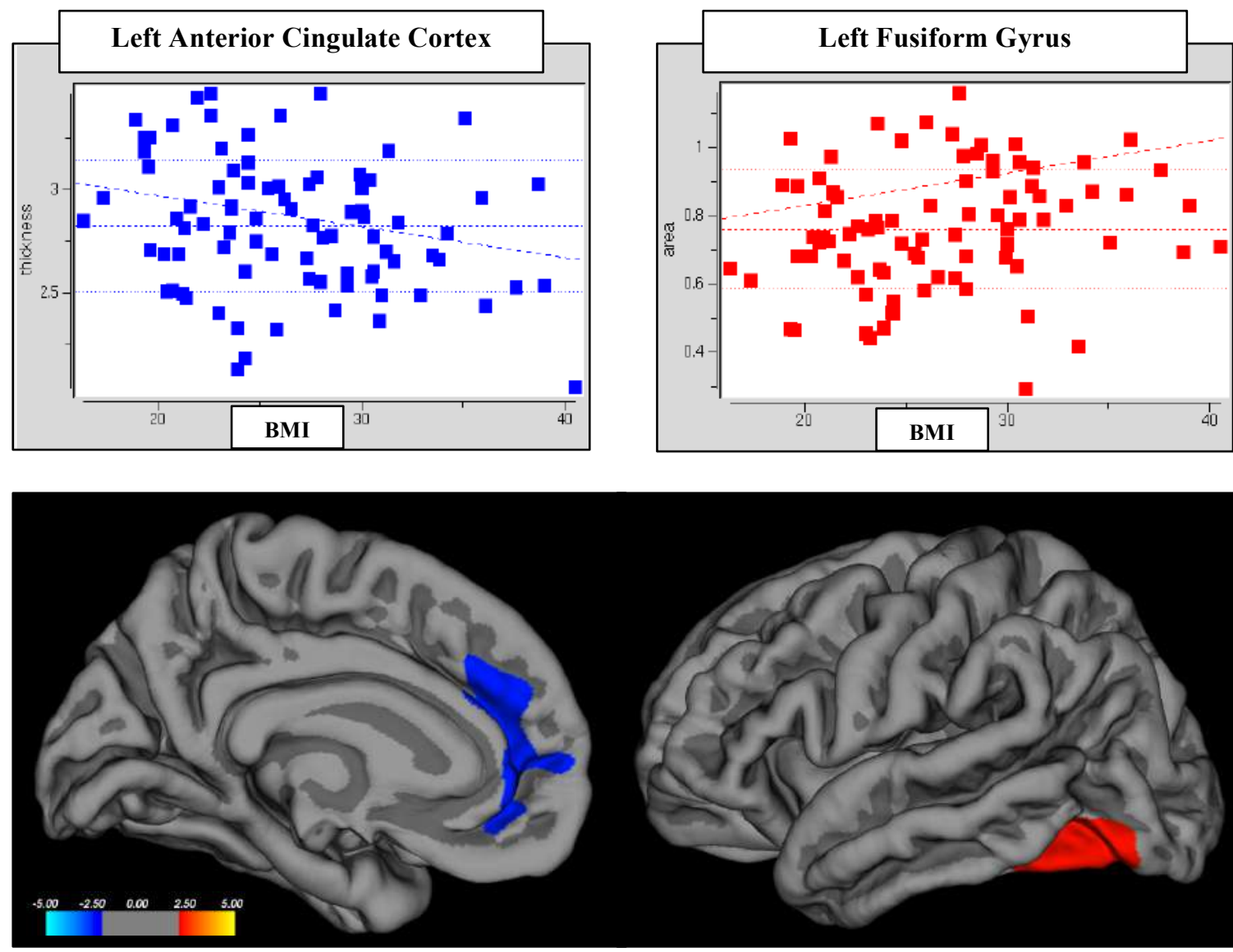

Figure 2: shows that increased BMI is associated with decreased cortical thickness in the left ACC and increased cortical surface area in the left fusiform gyrus 
Subcortical morphometry results showed that increased BMI related to increased bilateral globus pallidus and right amygdala volumes (Figure 3; Right Amygdala $r=$ $0.330, p=0.002$; Right Globus Pallidus $\mathrm{r}=0.268, p=0.015$; Left Globus Pallidus not shown; $\mathrm{r}=0.349, p=0.001$ ), indicating that increased body mass related to increased size of subcortical structures.

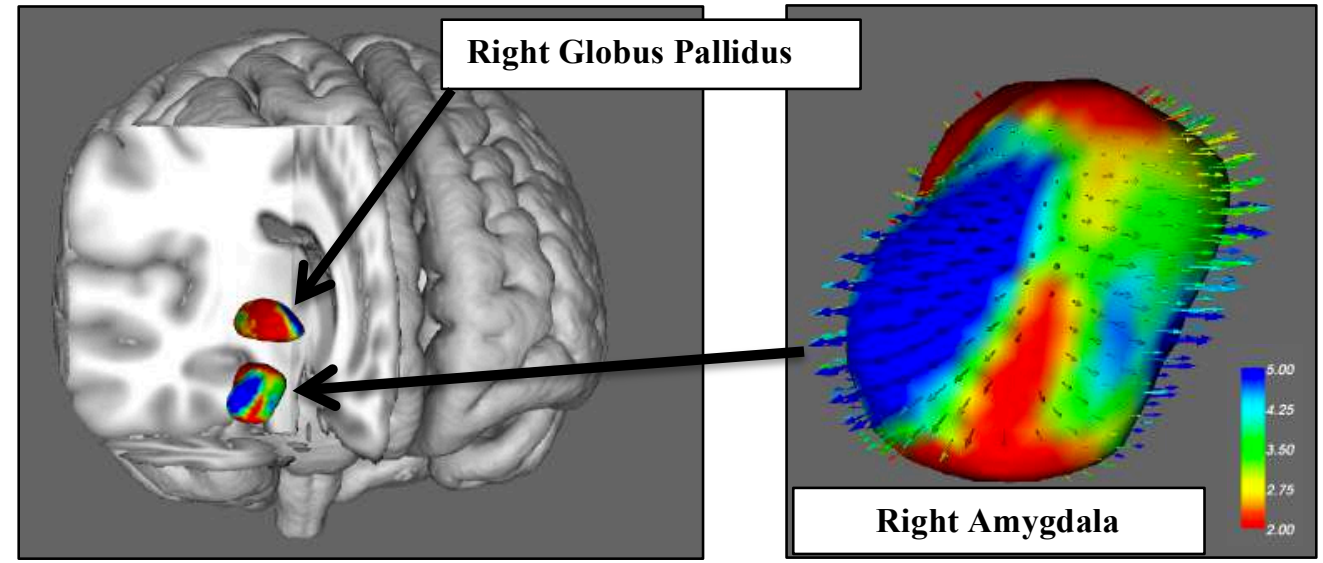

Figure 3: shows the spatial location of the amygdala and globus pallidus in the right hemisphere, as well as a closer look at the specific vertex changes of the right amygdala when assessed with BMI. The right amygdala and bilateral globus pallidus showed significant shape differences and increased volume with increased BMI.

\section{TBSS White Matter Analysis}

Examination of indicators of white matter integrity showed that increased BMI predicted increased FA in the left uncinate fasciculus (Figure 4), indicating increased body mass related to increased structural integrity of the uncinate fasciculus. The uncinate fasciculus connects subcortical structures, such as the amygdala, with ventral frontal cortical regions, including the subgenual ACC and vmPFC. 


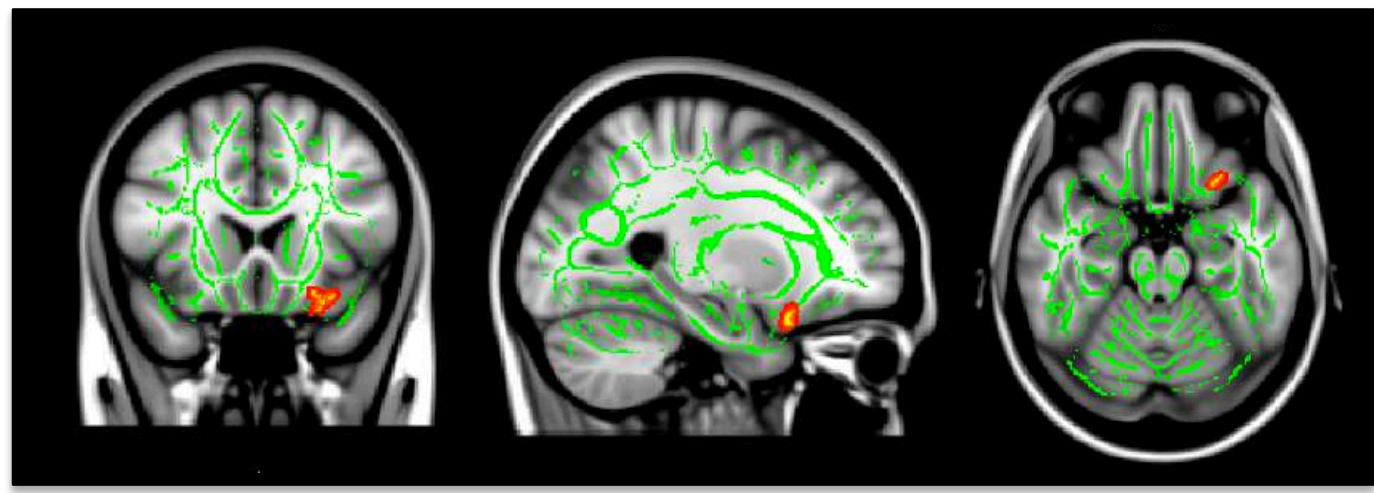

Figure 4: provided from left to right are coronal, sagittal, and axial views of a standard anatomical brain image. The average white matter skeleton for all subjects is shown in green, with the highlighted red area in the left hemisphere indicating a significant increase of FA in the uncinate fasciculus with increased BMI $(p<0.02)$.

\section{Wechsler Abbreviated Scale of Intelligence (WASI) Results}

\section{Surface-Based Morphometry (SBM)}

Cortical grey matter morphometry results showed that increased scores on WASI subtests related to broad increases in thickness and volume of frontal, parietal and visual cortex. The block design and similarities tests predicted increased cortical thickness and volume in the left superior parietal lobule and secondary visual (prestriate) cortex, whereas increased block design and vocabulary scores also predicted increased volume in the left inferior frontal gyrus (parsorbitalis) and left frontal pole (Figure 5). 

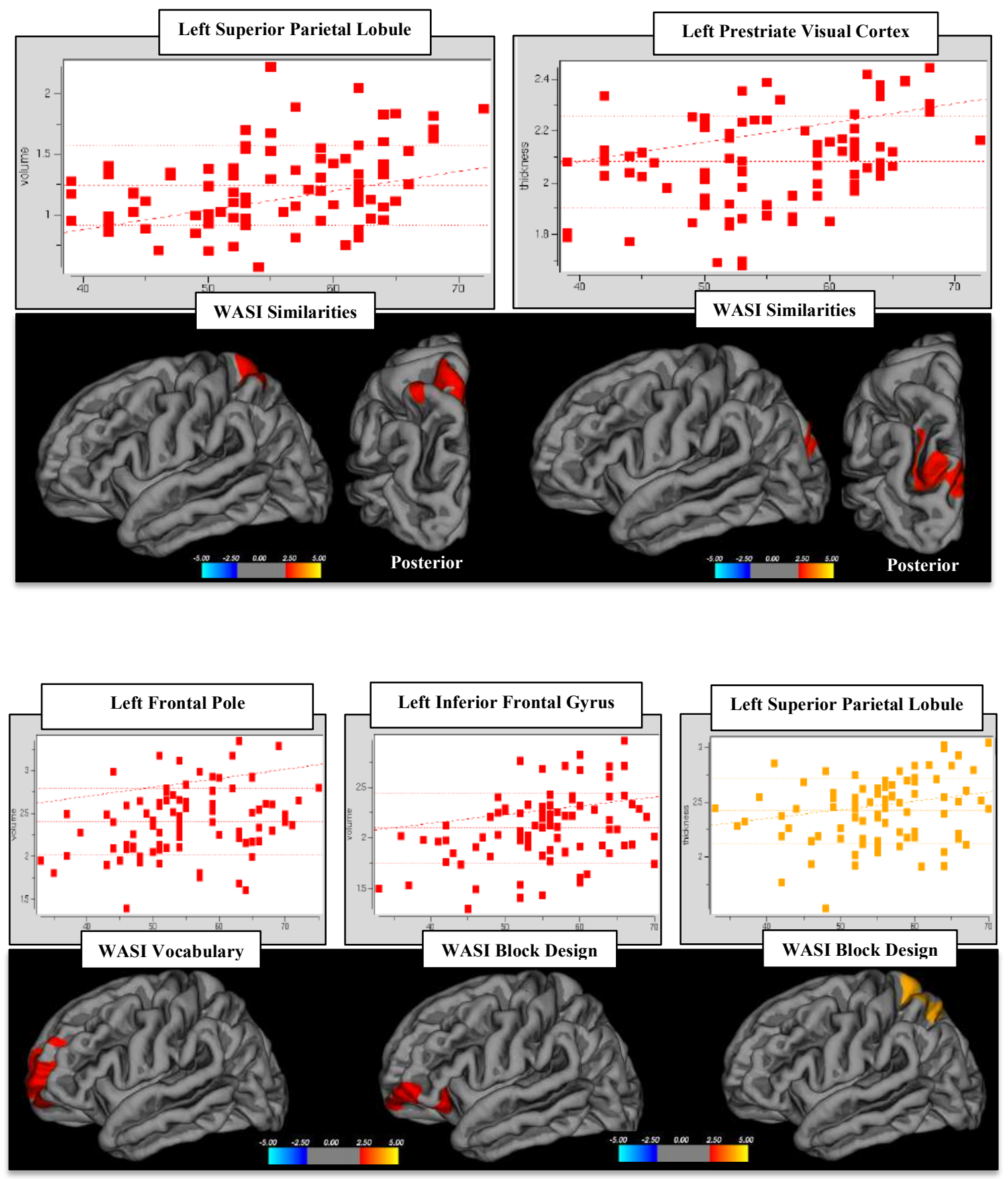

Figure 5: shows that increased WASI similarities scores related to increased volume in the left superior parietal lobule and also increased thickness in the left visual cortex. Whereas, increased vocabulary scores predicted increased volume in the left frontal pole and increased block design scores predicted increased volume in the left inferior frontal gyrus and increased thickness in the left superior parietal lobule. 


\section{Resting-State Functional Activation}

To assess functional attributes related to BMI and WASI, we examined resting state functional connectivity using ICA. Results indicated that similarities and vocabulary subtest scores showed positive relationships with certain brain regions associated with a functional connectivity component representing the default mode network (DMN). This suggests that subjects with higher intelligence scores were more likely to represent certain regions of this functional network. Specifically, increased intelligence scores predicted increased activation of the supplementary motor (SMA), motor and somatosensory areas, as well as the bilateral posterior hippocampal complex (Figure 6; Similarities $p<0.02$; Vocabulary $p<0.03$ ).

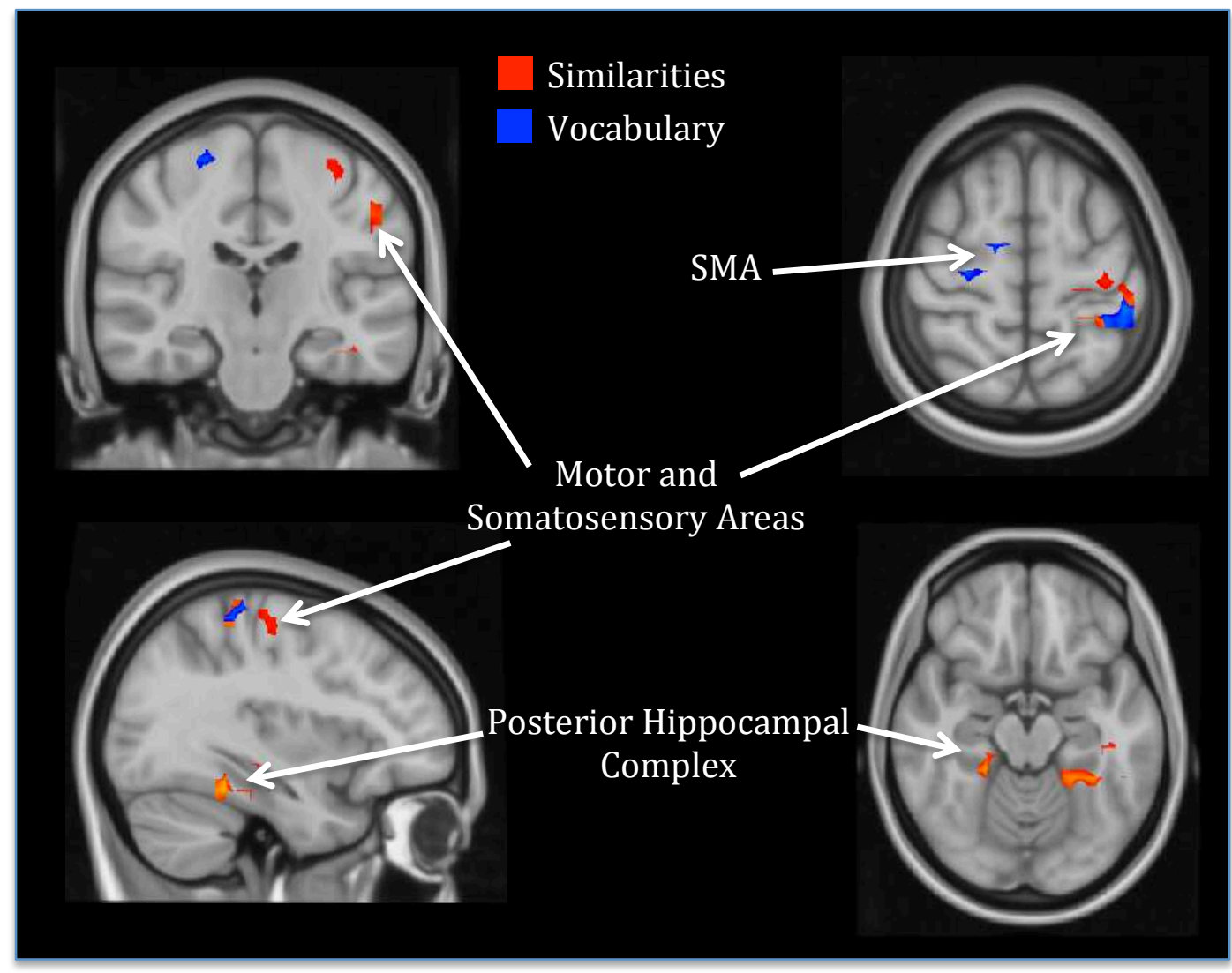

Figure 6: shows significant activation of areas associated with the WASI similarities (red) and vocabulary (blue) subtests. These include the supplementary motor area (SMA), motor and somatosensory areas, and the posterior hippocampal complex. 


\section{Discussion}

This study was the first, to our knowledge, to investigate the relationship between BMI and intelligence via a neural connection by examining cortical, subcortical and white matter structural integrity, as well as resting-state functional activation. Such a comprehensive cortical and subcortical morphometric structural and functional connectivity approach allowed for a deeper understanding of the neural mechanisms that underlie a mind-body relationship. Behaviorally, BMI and intelligence were negatively correlated, such that increased BMI predicted decreased WASI scores. Regarding their relationship with structural brain features, both measures seemed to relate primarily with frontal cortical gray matter regions, albeit in opposite directions. Surprisingly, there were few functional results from the resting-state data that related to these structural findings, suggesting that task-based functional imaging during an intelligence MR task may be needed to further clarify the observed effects on grey and white matter structures and their relationship to BMI and cognition. Nonetheless, the study findings do present evidence for neural factors that may be responsible for the relationship between BMI and intelligence, while also suggesting other measures of cognitive functioning that may be mediating these effects.

\section{$B M I$}

The structural results relating to BMI revealed morphometric alterations in a network that consists of the ACC, uncinate fasciculus (UF), and amygdala. Although there were hemispheric differences in these findings - left ACC and left UF in contrast to the right amygdala - these three structures nonetheless comprise a frontolimbic network that is involved in regulating emotion and reward processing (Bush et al. 2000; Botvinick 
et al., 2004; Etkin et al. 2006; Petrides \& Pandya, 2007; Ghashghaei et al., 2007; Wang et al., 2009). More specifically, the ACC and subgenual regions that are considered the vmPFC send projections to the amygdala through the UF. These projections are considered to form control afferents that ultimately provide inhibitory control over emotional response (Depue et al., 2015).

Interestingly, BMI has been linked with both impulsive behavior and a variety of emotional effects on eating behavior. Previous research shows that a positive relationship exists between BMI and emotion-driven impulsivity among adolescents, such that increases in BMI predict higher levels of both positive and negative urgency (DelgadoRico et al., 2012). Furthermore, higher BMI is positively linked to appetitive behaviors that include food responsiveness and emotional over-eating (Hunot et al., 2016). Similarly, overweight and obese individuals are also more likely to represent emotionand stress-related eating habits (Ozier et al., 2008), as well as overeating during emotional periods (Davis et al., 2004). Changes in this frontolimbic network that incorporates the ACC, UF, and amygdala may ultimately help explain these differences in behavior.

It is unclear, however, the specifics to which these structural changes are related to the negative relationship between BMI and intelligence. Perhaps most relevant to understanding the negative correlation is our finding in the ACC, since the reduced thickness in this region also extended to parts of the vmPFC. These frontal cortical regions are highly involved in both decision-making (Kennerley \& Walton, 2011) and inhibitory control (Li \& Shinha, 2008; Munakata et al., 2011), both of which may influence intelligence in some manner. Decision-making processes show a positive 
relationship with general intelligence (Danner et al., 2011; Shivapour et al., 2012) and it is also argued that intellectual ability relies on inhibitory control to activate task-relevant information and suppress task-irrelevant information (Dempster, 1991). Therefore, thinning of the ACC may provide the first clue as to how increasing BMI ultimately relates to decreases in general intelligence, via effects on other measures of cognitive functioning.

Furthermore, our finding of increased surface area in the left fusiform gyrus with increased BMI is rather interesting, given that previous studies also show a negative relationship between BMI and volume in this same region (Taki et al., 2008; Walther et al., 2010). This may suggest that the decreasing volume is accompanied by a general increase in surface area. Though the fusiform gyrus is most notable for its role in facial recognition (McCarthy et al., 1997), it has also been shown to be involved with networks that orient attention (Hopfinger et al., 2000) and also specifically direct attention to food stimuli (LaBar et al., 2001; Uher et al., 2006). Interestingly, high BMI does predict a greater attentional bias for food stimuli and switching attention to the most appetizing food (Yokum et. al. 2011). Increasing BMI therefore seems to be related to differences in a visual-attentional system, which may also mediate the negative relationship between BMI and intelligence.

There are certainly limitations with the results of this study, especially regarding the use of BMI as a body measurement. The common critique of BMI is its indirect measure of internal body physiology and therefore cannot be used as a direct assessment of physical health. Critical findings point towards BMI not accurately assessing certain populations, such as highly athletic individuals who are in peak physical health yet would 
clinically classify as overweight or obese based on BMI (Prentice \& Jebb, 2001). For this reason, it is important to note that BMI does not necessarily indicate a particular health state for an individual, but instead provides a quick method for predicting the risk of developing specific diseases that accompany increased fat or stress on the body. Despite these criticisms, BMI correlates better with visceral fat area and insulin sensitivity when compared to body adiposity index (BAI), another popular indirect measure of body fat (Hung et al., 2012; Suchanek et al., 2012). Furthermore, BMI also shows positive correlations with cholesterol and blood pressure, emphasizing the importance of this measurement in assessing risk for the development of serious health complications (McAdams et al., 2007).

Nonetheless, future studies pursuing a brain-mind-body approach can certainly benefit from using more direct measures of body physiology. Additionally, since other measures of cognitive functioning may mediate the intelligence-BMI relationship, more consideration should be placed on also assessing measures such as attention or inhibitory control.

\section{Intelligence}

Our structural findings relating to intelligence were primarily localized to the left prefrontal and parietal cortices, which reflect brain regions involved in a fronto-parietal attentional network. This network is considered to be the largest neural network that elicits top-down control for executive function during goal directed behavior (Corbetta \& Shulman, 2002). Therefore, this finding supports a structural relationship between intelligence and attention, which is further validated by evidence showing a positive correlation between these two cognitive measures (Schweizer et. al., 2005). Lateralization 
to the left hemisphere for these structural relationships with intelligence also seems to be in line with previous research demonstrating the importance of the left hemisphere for verbal skill and spatial reasoning (Langdon \& Warrington, 2000; Vallesi 2012), which are tested for on the WASI.

Interestingly, these structural findings relating to increased intelligence only show increased thickness and volume in frontal and parietal regions, contrasting with the decreased ACC thickness found with BMI. Increases in thickness and volume in these prefrontal regions have been linked to greater executive function and performance on cognitive control tasks (Burzynska et al., 2012; Ehrlich et al., 2012; Yuan \& Raz, 2014). This again points to a differential relationship that BMI and intelligence may have with regions involved in attention and decision-making.

Finally, resting-state analysis showed that increased WASI scores (vocabulary and similarities) predicted increased recruitment of a component representing the default mode network. Within this component, however, the positive correlation was primarily driven by increased activation in the pre-SMA, primary motor and somatosensory cortex, as well as the posterior hippocampal complex. These findings are not surprising, as sensory-motor integration is a major component of top-down attentional control linking cognition to behavioral output. Furthermore, the posterior hippocampus is specifically known to be involved with working memory and conceptual representations (Fanselow \& Dong, 2010; Sheldon \& Levine, 2016), which are cognitive abilities necessary for high performance on an intelligence test. 


\section{Conclusion}

Taken together, these results primarily indicate that BMI and intelligence are behaviorally anticorrelated, yet mediated by separate neuroanatomical substrates. Furthermore, the BMI-brain and intelligence-brain findings both suggest an attentional and decision-making system at play that might be mediating the BMI-intelligence relationship. Increased BMI was related to significant changes in a frontolimbic network consisting of the ACC and also cortical changes in the fusiform gyrus, suggesting effects on inhibitory control and attention. Increased WASI scores, however, predicted broad increases of cortical thickness and volume of a fronto-parietal attentional network, as well as functional recruitment of regions involved within this same network and workingmemory processing. Considering previous research and the present study, increased BMI may very well have a negative influence on cortical structure and subsequently cause decreases in a wide variety of cognitive functioning abilities, including intelligence. However, decreased intelligence may also adversely affect attention, such that individuals have a more difficult time resisting/inhibiting the temptation of food, which may then lead to BMI increases. Thus, the casual nature of this negative relationship between BMI and intelligence still remains unclear. 


\section{Acknowledgements}

The collaborative data-sharing initiative of the Nathan Kline Institute and the 1000 Functional Connectomes Project made this work possible. Additionally, I would like to thank my mentor, Dr. Brendan Depue, for his support and patience in the development of this thesis. I am grateful for the time he used to guide me through the process, as well as share his knowledge and expertise in neuroimaging and cognitive neuroscience. As well, I'd like to thank Kathryn Mattingly for her assistance in running the neural analyses. 


\section{References}

Andersson J.L.R., Jenkinson M. and Smith S. Non-linear optimisation. FMRIB technical report TR07JA1 from www.fmrib.ox.ac.uk/analysis/techrep

Andersson J.L.R., Jenkinson M. and Smith S. Non-linear registration, aka Spatial normalisation FMRIB technical report TR07JA2 from www.fmrib.ox.ac.uk/analysis/techrep

Au, J., Sheehan, E., Tsai, N., Duncan, G. J., Buschkuehl, M., \& Jaeggi, S. M. (2015). Improving fluid intelligence with training on working memory: a metaanalysis. Psychonomic Bulletin \& Review, 22(2), 366-377. doi:10.3758/s13423-014-0699-x

Beckmann C.F., Mackay C.E., Filippini N., and Smith S.M. Group comparison of resting-state FMRI data using multi-subject ICA and dual regression. OHBM, 2009.

Beckmann, C., \& Smith, S. (2004). Probabilistic Independent Component Analysis for Functional Magnetic Resonance Imaging. IEEE Transactions on Medical Imaging, 23(2), 137-152. doi:10.1109/tmi.2003.822821

Blond, B. N., Fredericks, C. A., \& Blumberg, H. P. (2012). Functional neuroanatomy of bipolar disorder: structure, function, and connectivity in an amygdalaanterior paralimbic neural system. Bipolar Disorders, 14(4), 340-355. doi:10.1111/j.1399-5618.2012.01015.x

Botvinick, M. M., Cohen, J. D., \& Carter, C. S. (2004). Conflict monitoring and anterior cingulate cortex: an update. Trends in Cognitive Sciences, 8(12), 539-546. doi:10.1016/j.tics.2004.10.003

Boyle, C. P., Raji, C. A., Erickson, K. I., Lopez, O. L., Becker, J. T., Gach, H. M., ... Thompson, P. M. (2015, January). Physical activity, body mass index, and brain atrophy in Alzheimer's disease. Neurobiology of Aging, 36. doi:10.1016/j.neurobiolaging.2014.05.036

Burzynska, A. Z., Nagel, I. E., Preuschhof, C., Gluth, S., Bäckman, L., Li, S., ... Heekeren, H. R. (2012). Cortical thickness is linked to executive functioning in adulthood and aging. Human Brain Mapping,33(7), 1607-1620. doi:10.1002/hbm.21311

Bush, G., Luu, P., \& Posner, M. I. (2000). Cognitive and emotional influences in anterior cingulate cortex. Trends in Cognitive Sciences, 4(6), 215-222. doi:10.1016/s1364-6613(00)01483-2

Corbetta, M., \& Shulman, G. L. (2002). Control Of Goal-Directed And Stimulus-Driven Attention In The Brain. Nature Reviews Neuroscience, 3(3), 201-215. doi:10.1038/nrn755

Dale, A. M., \& Sereno, M. I. (1993). Improved Localizadon of Cortical Activity by Combining EEG and MEG with MRI Cortical Surface Reconstruction: A Linear 
Approach. Journal of Cognitive Neuroscience, 5(2), 162-176.

http://doi.org/10.1162/jocn.1993.5.2.162

Dale, A. M., Fischl, B., \& Sereno, M. I. (1999). Cortical Surface-Based Analysis: I. Segmentation and Surface Reconstruction. NeuroImage, 9(2), 179-194. doi:10.1006/nimg.1998.0395

Danner, D., Hagemann, D., Schankin, A., Hager, M., \& Funke, J. (2011). Beyond IQ: A latent state-trait analysis of general intelligence, dynamic decision making, and implicit learning. Intelligence, 39(5), 323-334.

doi:10.1016/j.intell.2011.06.004

Davis, C., Levitan, R. D., Muglia, P., Bewell, C., \& Kennedy, J. L. (2004). DecisionMaking Deficits and Overeating: A Risk Model for Obesity. Obesity Research, 12(6), 929-935. doi:10.1038/oby.2004.113

Dempster, F. N. (1991). Inhibitory processes: A negleted dimension of intelligence. Intelligence, 15(2),157-173.doi:10.1016/0160-2896(91)90028-c

Depue, B. E., Orr, J. M., Smolker, H. R., Naaz, F., \& Banich, M. T. (2015). The Organization of Right Prefrontal Networks Reveals Common Mechanisms of Inhibitory Regulation Across Cognitive, Emotional, and Motor Processes. Cerebral Cortex, 26(4), 1634-1646. doi:10.1093/cercor/bhu324

Delgado-Rico, E., Río-Valle, J. S., González-Jiménez, E., Campoy, C., \& Verdejo-García, A. (2012). BMI Predicts Emotion-Driven Impulsivity and Cognitive Inflexibility in Adolescents With Excess Weight. Obesity, 20(8), 1604-1610. doi:10.1038/oby.2012.47

Desikan, R. S., Ségonne, F., Fischl, B., Quinn, B. T., Dickerson, B. C., Blacker, D., ... Killiany, R. J. (2006). An automated labeling system for subdividing the human cerebral cortex on MRI scans into gyral based regions of interest. NeuroImage, 31(3), 968-980. doi:10.1016/j.neuroimage.2006.01.021

Ehrlich, S., Brauns, S., Yendiki, A., Ho, B., Calhoun, V., Schulz, S. C., ... Sponheim, S. R. (2012). Associations of Cortical Thickness and Cognition in Patients With Schizophrenia and Healthy Controls. Schizophrenia Bulletin, 38(5), 10501062. doi:10.1093/schbul/sbr018

Etkin, A., Egner, T., Peraza, D. M., Kandel, E. R., \& Hirsch, J. (2006). Resolving Emotional Conflict: A Role for the Rostral Anterior Cingulate Cortex in Modulating Activity in the Amygdala. Neuron, 52(6), 1121. doi:10.1016/j.neuron.2006.12.003

Fanselow, M. S., \& Dong, H. (2010). Are the Dorsal and Ventral Hippocampus Functionally Distinct Structures? Neuron, 65(1), 7-19. doi:10.1016/j.neuron.2009.11.031

Filippini, N., Macintosh, B., Hough, M., Goodwin, G., Frisoni, G., Ebmeier, K., . . . Mackay, C. (2009). Distinct patterns of brain activity in young carriers of the APOE e4 allele. NeuroImage, 47. doi:10.1016/s1053-8119(09)71381-3 
Fischl, B., Sereno, M. I., \& Dale, A. M. (1999). Cortical Surface-Based Analysis: II: Inflation, Flattening, and a Surface-Based Coordinate System. NeuroImage, 9(2), 195-207. doi:10.1006/nimg.1998.0396

Fischl, B., Sereno, M. I., Tootell, R. B. H., \& Dale, A. M. (1999). High-Resolution Intersubject Averaging and a Coordinate System for the Cortical Surface. Human Brain Mapping, 8, 272-284.

Fischl, B., \& Dale, A. M. (2000). Measuring the thickness of the human cerebral cortex from magnetic resonance images. Proceedings of the National Academy of Sciences of the United States of America, 97(20), 11050-5. doi:10.1073/pnas.200033797

Fischl, B., Liu, A., \& Dale, A. M. (2001). Automated manifold surgery: constructing geometrically accurate and topologically correct models of the human cerebral cortex. IEEE Transactions on Medical Imaging, 20(1), 70-80. doi:10.1109/42.906426

Fischl, B., van der Kouwe, A., Destrieux, C., Halgren, E., Ségonne, F., Salat, D. H., ... Dale, A. M. (2004). Automatically parcellating the human cerebral cortex. Cerebral Cortex (New York, N.Y. : 1991), 14(1), 11-22. doi:10.1093/CERCOR/BHG087

Gignac, G., Vernon, P. A., \& Wickett, J. C. (2003). Factors Influencing the Relationship Between Brain Size and Intelligence. The Scientific Study of General Intelligence, 93-106. doi:10.1016/b978-008043793-4/50042-8

Ghashghaei, H., Hilgetag, C., \& Barbas, H. (2007). Sequence of information processing for emotions based on the anatomic dialogue between prefrontal cortex and amygdala. NeuroImage, 34(3), 905-923. doi:10.1016/j.neuroimage.2006.09.046

Greenberg, A. S., \& Obin, M. S. (2006). Obesity and the role of adipose tissue in inflammation and metabolism. The American Journal of Clinical Nutrition, 83(2), 461S-465S.

Gunstad, J., Paul, R. H., Cohen, R. A., Tate, D. F., Spitznagel, M. B., \& Gordon, E. (2007). Elevated body mass index is associated with executive dysfunction in otherwise healthy adults. Comprehensive Psychiatry, 48(1), 57-61. doi:10.1016/j.comppsych.2006.05.001

Gunstad, J., Paul, R. H., Cohen, R. A., Tate, D. F., Spitznagel, M. B., Grieve, S., \& Gordon, E. (2008). Relationship Between Body Mass Index and Brain Volume in Healthy Adults. International Journal of Neuroscience, 118(11), 1582-1593. doi:10.1080/00207450701392282

Gottfredson, L. S. (1998). The General Intelligence Factor. Scientific American Presents, 9(4), 24-29.

Haier, R. J., Jung, R. E., Yeo, R. A., Head, K., \& Alkire, M. T. (2004). Structural brain variation and general intelligence. NeuroImage, 23(1), 425-433. doi:10.1016/j.neuroimage.2004.04.025 
Hassenstab, J. J., Sweet, L. H., Parigi, A. D., Mccaffery, J. M., Haley, A. P., Demos, K. E., .. .Wing, R. R. (2012). Cortical thickness of the cognitive control network in obesity and successful weight loss maintenance: A preliminary MRI study. Psychiatry Research: Neuroimaging, 202(1), 77-79.

doi:10.1016/j.pscychresns.2011.09.008

Hopfinger, J. B., Buonocore, M. H., \& Mangun, G. R. (2000). The neural mechanisms of top- down attentional control. Nature Neuroscience , 3(3), 284-291. doi:10.1038/72999

Hung, C., Yang, C., Hsieh, H., Wei, J., Ma, W., \& Li, H. (2012). BMI Correlates Better to Visceral Fat and Insulin Sensitivity Than BAI. Obesity, 20(6), 1141. doi:10.1038/oby.2012.86

Hunot, C., Fildes, A., Croker, H., Llewellyn, C. H., Wardle, J., \& Beeken, R. J. (2016). Appetitive traits and relationships with BMI in adults: Development of the Adult Eating Behaviour Questionnaire. Appetite, 105, 356-363. doi:10.1016/j.appet.2016.05.024

Jaeggi, S. M., Buschkuehl, M., Jonides, J., \& Perrig, W. J. (2008). Improving fluid intelligence with training on working memory. Proceedings of the National Academy of Sciences, 105(19), 6829-6833. doi:10.1073/pnas.0801268105

Kanazawa, S. (2014). Intelligence and obesity. Current Opinion in Endocrinology \& Diabetes and Obesity, 21(5), 339-344. doi:10.1097/med.0000000000000091

Kennerley, S. W., \& Walton, M. E. (2011). Decision making and reward in frontal cortex: Complementary evidence from neurophysiological and neuropsychological studies. Behavioral Neuroscience, 125(3), 297-317. doi:10.1037/a0023575

Keys, A., Fidanza, F., Karvonen, M. J., Kimura, N., \& Taylor, H. L. (1972). Indices of relative weight and obesity. Journal of Chronic Diseases, 25(6-7), 329-343. doi:10.1016/0021-9681(72)90027-6

Kopelman, P. (2007). Health risks associated with overweight and obesity. Obesity Reviews, 8(S1), 13-17. doi:10.1111/j.1467-789x.2007.00311.x

Kullmann, S., Heni, M., Veit, R., Ketterer, C., Schick, F., Häring, H., . . Preissl, H. (2012). The obese brain: Association of body mass index and insulin sensitivity with resting state network functional connectivity. Human Brain Mapping, 33(5), 1052-1061. doi:10.1002/hbm.21268

Langdon, D., \& Warrington, E. K. (2000). The Role of the Left Hemisphere in Verbal and Spatial Reasoning Tasks. Cortex, 36(5), 691-702. doi:10.1016/s00109452(08)70546-x

Lavagnino, L., Mwangi, B., Bauer, I. E., Cao, B., Selvaraj, S., Prossin, A., \& Soares, J. C. (2016). Reduced Inhibitory Control Mediates the Relationship Between Cortical Thickness in the Right Superior Frontal Gyrus and Body Mass Index. Neuropsychopharmacology, 41(9), 2275-2282. doi:10.1038/npp.2016.26 
Lee, B. C., \& Lee, J. (2014). Cellular and molecular players in adipose tissue inflammation in the development of obesity-induced insulin resistance. $B B A$ Molecular Basis of Disease, 1842(3), 446-462. doi:10.1016/j.bbadis.2013.05.017

Li, C. R., \& Sinha, R. (2008). Inhibitory control and emotional stress regulation: Neuroimaging evidence for frontal-limbic dysfunction in psycho-stimulant addiction. Neuroscience \& Biobehavioral Reviews, 32(3), 581-597. doi:10.1016/j.neubiorev.2007.10.003

Lips, M. A., Wijngaarden, M. A., Grond, J. V., Buchem, M. A., Groot, G. H., Rombouts, S. A., ... Veer, I. M. (2014). Resting-state functional connectivity of brain regions involved in cognitive control, motivation, and reward is enhanced in obese females. American Journal of Clinical Nutrition, 100(2), 524-531. doi:10.3945/ajcn.113.080671

Labar, K. S., Gitelman, D. R., Parrish, T. B., Kim, Y., Nobre, A. C., \& Mesulam, M. -. (2001). Hunger selectively modulates corticolimbic activation to food stimuli in humans. Behavioral Neuroscience, 115(2), 493-500. doi:10.1037//07357044.115.2.493

Marqués-Iturria, I., Pueyo, R., Garolera, M., Segura, B., Junqué, C., García-García, I., . . . Jurado, M. Á. (2013). Frontal cortical thinning and subcortical volume reductions in early adulthood obesity. Psychiatry Research: Neuroimaging, 214(2), 109-115. doi:10.1016/j.pscychresns.2013.06.004

Mcadams, M. A., Van Dam, R. M., \& Hu, F. B. (2007). Comparison of Self-reported and Measured BMI as Correlates of Disease Markers in U.S. Adults*. Obesity, 15(1), 188-196. doi:10.1038/oby.2007.504

Mccarthy, G., Puce, A., Gore, J. C., \& Allison, T. (1997). Face-Specific Processing in the Human Fusiform Gyrus. Journal of Cognitive Neuroscience, 9(5), 605-610. doi:10.1162/jocn.1997.9.5.605

Medic, N., Ziauddeen, H., Ersche, K. D., Farooqi, I. S., Bullmore, E. T., Nathan, P. J., . . . Fletcher, P. C. (2016). Increased body mass index is associated with specific regional alterations in brain structure. International Journal of Obesity, 40(7), 1177-1182. doi:10.1038/ijo.2016.42

Menary, K., Collins, P. F., Porter, J. N., Muetzel, R., Olson, E. A., Kumar, V., . . Luciana, M. (2013). Associations between cortical thickness and general intelligence in children, adolescents and young adults. Intelligence, 41(5), 597-606. doi:10.1016/j.intell.2013.07.010

Mokdad, A. H., Ford, E. S., Bowman, B. A., Dietz, W. H., Vinicor, F., Bales, V. S., \& Marks, J. S. (2003). Prevalence of Obesity, Diabetes, and Obesity-Related Health Risk Factors, 2001. Jama, 289(1), 76-79. doi:10.1001/jama.289.1.76

Morey, R. A., Petty, C. M., Xu, Y., Pannu Hayes, J., Wagner, H. R., Lewis, D. V., ... McCarthy, G. (2009). A comparison of automated segmentation and manual 
tracing for quantifying hippocampal and amygdala volumes. NeuroImage, 45(3), 855-866. doi:10.1016/j.neuroimage.2008.12.033

Munakata, Y., Herd, S. A., Chatham, C. H., Depue, B. E., Banich, M. T., \& O'Reilly, R. C. (2011). A unified framework for inhibitory control. Trends in Cognitive Sciences, 15(10), 453-459. doi:10.1016/j.tics.2011.07.011

Neisser, U., Boodoo, G., Bouchard, T., Boykin, A. W., Brody, N., Ceci, S., ... Urbina, S. (1996). Intelligence: Knowns and Unknowns . American Psychologist, 51(2), 77-101. doi:10.1037/0003-066X.51.2.77

Shivapour, S. K., Nguyen, C. M., Cole, C. A., \& Denburg, N. L. (2012). Effects of Age, Sex, and Neuropsychological Performance on Financial DecisionMaking. Frontiers in Neuroscience, 6. doi:10.3389/fnins.2012.00082

Smith, S. M. (2002). Fast robust automated brain extraction. Human Brain Mapping, 17(3), 143-155. doi:10.1002/hbm.10062

Smith, S. M., Jenkinson, M., Woolrich, M. W., Beckmann, C. F., Behrens, T. E., Johansen-Berg, H., ... Matthews, P. M. (2004). Advances in functional and structural MR image analysis and implementation as FSL. NeuroImage, 23. doi:10.1016/j.neuroimage.2004.07.051

Smith, S. M., Jenkinson, M., Johansen-Berg, H., Rueckert, D., Nichols, T. E., Mackay, C. E., ... Behrens, T. E. (2006). Tract-based spatial statistics: Voxelwise analysis of multi-subject diffusion data. NeuroImage, 31(4), 1487-1505. doi:10.1016/j.neuroimage.2006.02.024

Spearman, C. (1904). "General Intelligence," Objectively Determined and Measured. The American Journal of Psychology, 15(2), 201-293. doi:10.2307/1412107

Ozier, A. D., Kendrick, O. W., Leeper, J. D., Knol, L. L., Perko, M., \& Burnham, J. (2008). Overweight and Obesity Are Associated with Emotion- and Stress-Related Eating as Measured by the Eating and Appraisal Due to Emotions and Stress Questionnaire. Journal of the American Dietetic Association, 108(1), 49-56. doi:10.1016/j.jada.2007.10.011

Patenaude, B. (2007). Bayesian statistical models of shape and appearance for subcortical brain segmentation. Oxford, U.K.

Patenaude B., Smith S., Kennedy D., J. M. (2007). FIRST-FMRIB's integrated registration and segmentation tool. In Human Brain Mapping.

Patenaude B., Smith S., Kennedy D., J. M. (2008). Improved surface models for FIRST. In Human Brain Mapping.

Petrides, M., \& Pandya, D. N. (2007). Efferent Association Pathways from the Rostral Prefrontal Cortex in the Macaque Monkey. Journal of Neuroscience, 27(43), 11573-11586. doi:10.1523/jneurosci.2419-07.2007

Prentice, A. M., \& Jebb, S. A. (2001). Beyond body mass index. Obesity Reviews, 2(3), 141-147. doi:10.1046/j.1467-789x.2001.00031.x 
Reuter, M., Rosas, H. D., \& Fischl, B. (2010). Highly accurate inverse consistent registration: A robust approach. NeuroImage, 53(4), 1181-1196. doi:10.1016/j.neuroimage.2010.07.020

Roth, G., \& Dicke, U. (2005). Evolution of the brain and intelligence. Trends in Cognitive Sciences, 9(5), 250-257. doi:10.1016/j.tics.2005.03.005

Rueckert, D., Sonoda, L., Hayes, C., Hill, D., Leach, M., \& Hawkes, D. (1999). Nonrigid registration using free-form deformations: application to breast MR images. IEEE Transactions on Medical Imaging, 18(8), 712-721. doi:10.1109/42.796284

Schweizer, K., Moosbrugger, H., \& Goldhammer, F. (2005). The structure of the relationship between attention and intelligence. Intelligence, 33(6), 589-611. doi:10.1016/j.intell.2005.07.001

Ségonne, F., Dale, A. M., Busa, E., Glessner, M., Salat, D., Hahn, H. K., \& Fischl, B. (2004). A hybrid approach to the skull stripping problem in MRI. NeuroImage, 22(3), 1060-1075. doi:10.1016/j.neuroimage.2004.03.032

Segonne, F., Pacheco, J., \& Fischl, B. (2007). Geometrically Accurate TopologyCorrection of Cortical Surfaces Using Nonseparating Loops. IEEE Transactions on Medical Imaging, 26(4), 518-529. doi:10.1109/TMI.2006.887364

Sheldon, S., \& Levine, B. (2016). The role of the hippocampus in memory and mental construction. Annals of the New York Academy of Sciences, 1369(1), 76-92. doi:10.1111/nyas.13006

Sled, J. G., Zijdenbos, A. P., \& Evans, A. C. (1998). A nonparametric method for automatic correction of intensity nonuniformity in MRI data. IEEE Transactions on Medical Imaging, 17(1), 87-97. doi:10.1109/42.668698

Song, M., Zhou, Y., Li, J., Liu, Y., Tian, L., Yu, C., \& Jiang, T. (2008). Brain spontaneous functional connectivity and intelligence. NeuroImage, 41(3), 1168-1176. doi:10.1016/j.neuroimage.2008.02.036

Suchanek, P., Lesna-Kralova, I., Mengerova, O., Mrazkova, J., Lanska, V., \& Stavek, P. (2012). Which index best correlates with body fat mass: BAI, BMI, waist or WHR? Neuroendocrinology Letters, 33, 78-82.

Stanek, K. M., Grieve, S. M., Brickman, A. M., Korgaonkar, M. S., Paul, R. H., Cohen, R. A., \& Gunstad, J. J. (2011). Obesity Is Associated With Reduced White Matter Integrity in Otherwise Healthy Adults. Obesity, 19(3), 500-504.

doi:10.1038/oby.2010.312

Taki, Y., Kinomura, S., Sato, K., Inoue, K., Goto, R., Okada, K., . . Fukuda, H. (2008). Relationship Between Body Mass Index and Gray Matter Volume in 1,428 Healthy Individuals. Obesity, 16(1), 119-124. doi:10.1038/oby.2007.4 
Uher, R., Treasure, J., Heining, M., Brammer, M. J., \& Campbell, I. C. (2006). Cerebral processing of food-related stimuli: Effects of fasting and gender. Behavioural Brain Research, 169(1), 111-119. doi:10.1016/j.bbr.2005.12.008

Vallesi, A. (2012). Organisation of executive functions: Hemispheric asymmetries. Journal of Cognitive Psychology, 24(4), 367-386. doi:10.1080/20445911.2012.678992

Walther, K., Birdsill, A. C., Glisky, E. L., \& Ryan, L. (2010). Structural brain differences and cognitive functioning related to body mass index in older females. Human Brain Mapping, 31(7), 1052-1064. doi:10.1002/hbm.20916

Wechsler, D (2011). Wechsler Abbreviated Scale of Intelligence, Second Edition (WASI-II). San Antonio, TX:NCS Pearson. doi: 10.1177/0734282912467756

Winkler, A. M., Ridgway, G. R., Webster, M. A., Smith, S. M., \& Nichols, T. E. (2014). Permutation inference for the general linear model. NeuroImage, 92, 381397. doi:10.1016/j.neuroimage.2014.01.060

Woolrich, M. W., Jbabdi, S., Patenaude, B., Chappell, M., Makni, S., Behrens, T., ... Smith, S. M. (2009). Bayesian analysis of neuroimaging data in FSL. NeuroImage, 45(1), S173-S186. doi:10.1016/j.neuroimage.2008.10.055

Xu, J., Li, Y., Lin, H., Sinha, R., \& Potenza, M. N. (2013). Body mass index correlates negatively with white matter integrity in the fornix and corpus callosum: A diffusion tensor imaging study. Human Brain Mapping, 34(5), 1044-1052. doi:10.1002/hbm.21491

Yeo, B. T., Krienen, F. M., Sepulcre, J., Sabuncu, M. R., Lashkari, D., Hollinshead, M., ... Buckner, R. L. (2011). The organization of the human cerebral cortex estimated by intrinsic functional connectivity. Journal of Neurophysiology, 106(3), 1125-1165. doi:10.1152/jn.00338.201

Yokum, S., Ng, J., \& Stice, E. (2011). Attentional Bias to Food Images Associated With Elevated Weight and Future Weight Gain: An fMRI Study. Obesity, 19(9), 1775-1783. doi:10.1038/oby.2011.168

Yokum, S., Ng, J., \& Stice, E. (2012). Relation of regional gray and white matter volumes to current BMI and future increases in BMI: a prospective MRI study. International Journal of Obesity, 36(5), 656-664. doi:10.1038/ijo.2011.175

Yuan, P., \& Raz, N. (2014). Prefrontal cortex and executive functions in healthy adults: A meta-analysis of structural neuroimaging studies. Neuroscience \& Biobehavioral Reviews, 42, 180-192. doi:10.1016/j.neubiorev.2014.02.005 